\title{
¿JUGAMOS? MEJORA DE LA INTERACCIÓN HUMANO-ROBOT MEDIANTE JUEGOS DE MESA
}

\author{
Mercedes Garcia-Salguero, Javier Monroy, Javier Gonzalez-Jimenez \\ Dpto. Ingeniería de Sistemas y Automática, Instituto de Investigación Biomédica \\ de Málaga (IBIMA), Universidad de Málaga, Campus de Teatinos, 29071, Málaga \\ \{mercedesgarsal, jgmonroy, javiergonzalez\}@uma.es
}

\begin{abstract}
Resumen
En el contexto de un robot de servicio o asistencial, y en relación a su interacción con un usuario, este trabajo presenta un sistema que permite al robot ofrecer y llevar a cabo partidas de juegos de mesa. El sistema propuesto, que busca ampliar el abanico de actividades que el robot puede compartir con el usuario, se centra en ofrecer una experiencia lo más similar posible a una partida tradicional entre dos personas. Para ello, el juego se proyecta sobre una mesa mediante un pequeño proyector montado sobre el robot, y un sistema de percepción visual es empleado para detectar los movimientos del usuario. El sistema propuesto es especialmente interesante para robots asistenciales en entornos residenciales con personas mayores, permitiendo al usuario desarrollar diferentes actividades beneficiosas para su salud (memoria, reflejos, coordinación, etc.). Para validar el sistema propuesto, se presenta un caso práctico con un robot real en un entorno de laboratorio.
\end{abstract}

Palabras clave: HRI, interacción robot-usuario, juegos de mesa, transformación proyectiva, ROS

\section{INTRODUCCIÓN}

Durante los últimos años, el envejecimiento poblacional y el aumento de la esperanza de vida asociado han obligado a atender con mayor frecuencia los problemas propios de edades avanzadas, entre ellos, la necesidad de una asistencia continua para el desarrollo de las tareas cotidianas y el preocupante deterioro cognitivo asociado a la senectud. Conscientes de esta realidad, la robótica asistencial se ha propuesto como una alternativa y solución para el cuidado de las personas mayores en sus propios hogares. Diversos son los proyectos europeos, como Excite [6], Teresa [8] o GiraffPlus [3], que proponen la fusión de entornos domóticos con plataformas móviles capaces de ofrecer diversos servicios a los usuarios $[5,4]$.

Por otro lado y tal y como apunta el estudio Ludiman [1] desarrollado conjuntamente por el instituto de biomecánica de Valencia y por el instituto tecnológico de producto infantil y ocio (AIJU), los

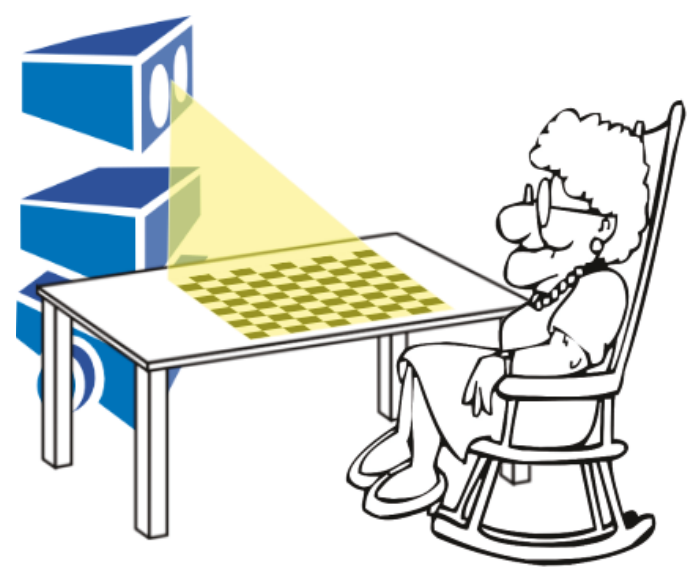

Figura 1: Ilustración de una partida robot-usuario mediante la plataforma de juegos propuesta. Un pequeño proyector integrado en el robot recrea el juego sobre una mesa mientras que un sistema de visión artificial capta los movimientos del usuario.

juegos de mesa pueden ser usados como una herramienta en pos de prevenir los efectos negativos en el cerebro derivados del envejecimiento, ayudando a mantener las capacidades cognitivas y físicas de las personas mayores. Los juegos mejoran la movilidad y la agilidad -ya que requieren coordinación-, incrementan la atención, estimulan la percepción sensorial y, en general, la necesidad de recordar las reglas del juego contribuye al mantenimiento de las habilidades cognitivas y de la memoria.

No obstante, para una persona mayor existen una serie de limitaciones físicas y tecnológicas que hacen que tanto los juegos tradicionales como los más modernos videojuegos no siempre sean una opción factible. Los primeros por el hecho de necesitar de tablero y piezas físicas, requiriendo de un espacio de almacenamiento, lo cual reduce la variedad de juegos disponibles, así como limitaciones físicas relacionadas con el pequeño tamaño de estas piezas. Solventando estos inconvenientes se hallarían los videojuegos, los cuales pueden ser accedidos desde tablets, smartphones o incluso desde el propio display del robot. No obstante, las habilidades tecnológicas de este sector de la población pueden suponer una barrera a la hora de jugar, haciendo que los videojuegos no siempre sean una opción interesante. 
Es por esto que implementar un sistema de juegos "mixto" que resulte sencillo de utilizar para una persona mayor supone un paso lógico. Además, el incorporarlo en un robot de asistencia supone una buena alternativa a los juegos tradicionales de mesa para mejorar y mantener las habilidades cognitivas. De esta forma, el robot asistente podría proponer a la persona mayor jugar a una hora específica del día, cada cierto tiempo o incluso la posibilidad de jugar online, lo cual contribuiría a mejorar las habilidades sociales del usuario. Así mismo, otras ventajas incluirían controlar el tiempo que se ha estado jugando y parar/posponer el juego en cualquier momento. A todo esto se suman las otras muchas habilidades propias del robot, las cuales estarían presentes antes, durante y después del juego, desde poder navegar o hablar con el usuario, hasta solicitar ayuda en caso de necesitarla.

En este trabajo se describe una plataforma que permite al robot relacionarse con el usuario mediante los juegos de mesa tradicionales. Dicho sistema se diseña atendiendo a la necesidad de su facilidad de uso, al tamaño físico del juego (preferiblemente grande) y a la variedad en los juegos a ofrecer. Teniendo en cuenta estas características, se propone implementar este sistema de juegos mediante un proyector que recreará el juego de mesa para el usuario y un sistema de percepción para detectar sus movimientos, procurando una interacción fluida entre robot y usuario. La Figura 1 ilustra el sistema propuesto, donde se aprecia como el usuario se sitúa frente al robot para jugar, manteniendo la posición habitual en este tipo de juegos "con contrincante".

La plataforma de juegos propuesta precisa de una serie de módulos que deben coordinarse para un correcto funcionamiento de la misma. Dichos módulos surgen de diversas necesidades relacionados con la naturaleza del sistema. Por una parte, la posición del sistema de visión sobre el robot provoca distorsiones que deben ser corregidas. Por otro lado, es necesario estimar los movimientos del usuario así como conjugar los elementos que intervienen durante la sesión de juegos. Por último, es necesario que el robot se mueva, aproximándose y alejándose de la mesa, antes y después de cada sesión.

Para finalizar, las Secciones de este trabajo se organizan de la siguiente forma: se comienza describiendo la arquitectura del sistema en la Sección 2 siguiendo con la interacción usuario-robot en la Sección 3, para a continuación definir la calibración del sistema en la Sección 4. Detalles de implementación relacionados con el motor de videojuegos y el sistema de movimiento del robot

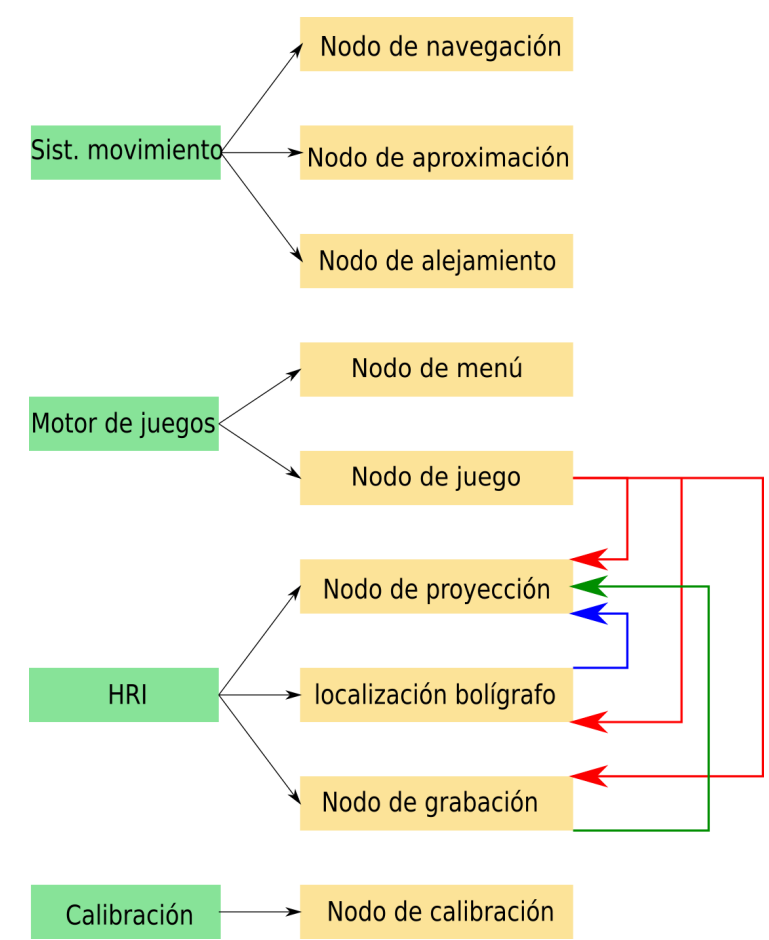

Figura 2: Diagrama de bloques del sistema propuesto. Los cuatro bloques principales (verde) están compuestos por uno o varios nodos ROS (naranja) que se comunican entre sí para permitir al robot jugar con el usuario.

se analizan en las Secciones 5 y 6, respectivamente, mientras que la Sección 7 introduce un caso práctico (prueba de concepto). Para finalizar, la Sección 8 describe las conclusiones y los trabajos futuros.

\section{DESCRIPCIÓN DEL SISTEMA}

El sistema propuesto consta de cuatro bloques principales (véase Figura 2): interacción humanorobot (usualmente conocida por sus siglas en inglés, HRI, human-robot interaction), calibración, motor de juegos y movimiento del robot. Cada uno de estos bloques se centra en un aspecto fundamental y necesario para permitir que un robot social pueda jugar con un usuario de una forma natural. Estos bloques se organizan sobre la ampliamente extendida arquitectura robótica ROS [7], la cual provee de los mecanismos de comunicación y coordinación para que diferentes bloques funcionales, denominados "nodos", puedan realizar sus tareas de forma eficiente.

De forma general, el flujo de acciones que el robot debe realizar a la hora de ofrecer jugar una partida al usuario viene definida por la siguiente secuencia: (i) navegación del robot hasta la mesa donde se llevará a cabo la partida y su posterior aproximación a esta, (ii) ejecución del motor de juegos ofreciendo inicialmente un menú con diver- 
sos juegos entre los que elegir y (iii) finalmente, tras indicación del usuario de su intención de terminar, el cierre del sistema y la consiguiente navegación del robot para alejarse de la mesa de juego. Es importante mencionar que el menú de juegos es una etapa recurrente que se muestra al inicio de la sesión y tras terminar cada partida, dando la posibilidad de jugar varias veces a un mismo juego o incluso a diferentes juegos en una misma sesión.

Descripción de la funcionalidad, así como detalles de implementación de cada uno de los cuatro bloques que componen el sistema son presentados en las siguientes Secciones.

\section{INTERACCIÓN ROBOT-USUARIO}

Esta Sección detalla la comunicación bidireccional entre el robot y el usuario, tomando ambos el rol de contrincantes en un juego de mesa. Por un lado se describe el sistema de proyección del juego así como el sistema de voz empleado para reforzar la comunicación robot-usuario, y por otro lado se analiza el sistema de percepción empleado para estimar los movimientos del usuario en lo referente a la partida en juego.

\subsection{Proyección del Juego}

La proyección del tablero y las piezas sobre la mesa permite al usuario poder jugar al juego que está siendo ejecutado en el robot, pero de una manera sencilla y cómoda, semejante a la disposición de los juegos de mesa tradicionales. No obstante, para que la proyección del juego sea lo más fidedigna y amigable posible para el jugador, es necesario tener en cuenta una serie de aspectos técnicos:

- Transformación Proyectiva: La proyección del juego sobre el plano de la mesa (véase Figura 3) conlleva una deformación en la imagen fruto de la posición relativa entre el proyector y la superficie de la mesa. Para compensar dicho efecto es necesario pre-procesar la imagen a mostrar aplicándole la transformación proyectiva detallada en la Sección 4.

- Rotación de la Imagen: Dado el rol de contrincante del robot a la hora de jugar, es necesario rotar la imagen a mostrar $180^{\circ}$ para que el usuario, que se sienta en frente, la vea con su orientación original.

- Opción de Cierre: Como en toda aplicación, es necesario proveer al usuario con la opción de salir del juego. Por ello se añade, sobre el juego mostrado, un botón rojo en la esquina inferior derecha.

- Puntero Señalador: Con el objetivo de facilitar la interacción con el usuario, durante una partida activa se muestra la localización del puntero como un pequeño círculo azul. Este puntero hace referencia al dispositivo señalador utilizado para detectar los movimientos del usuario, el cual es detallado en la Sección 3.2 .

\subsubsection{Comunicación por Voz con el Usuario}

Otra de las formas de interacción con el usuario utilizadas es mediante voz. Se han añadido frases en cada etapa del juego con el fin de hacer más inmersiva la interacción del robot con el humano. De esta forma, los estímulos sensoriales que el usuario percibe durante el juego se alternan en visuales y auditivos, eliminando por otra parte los momentos de silencio que puedan ocurrir en los distintos pasos del proceso del juego, como la espera hasta que este es lanzado.

No obstante y en el momento en el cual tiene lugar este proyecto, la comunicación por voz comentada es unidireccional, produciéndose únicamente desde el robot hacia el jugador.

\subsection{Sistema de Percepción}

Dado que el juego es presentado al usuario proyectándolo sobre una mesa, es necesario emplear un sistema de percepción que permita discernir los movimientos del usuario durante la partida. Para ello, se ha optado por emplear un bolígrafo tipo

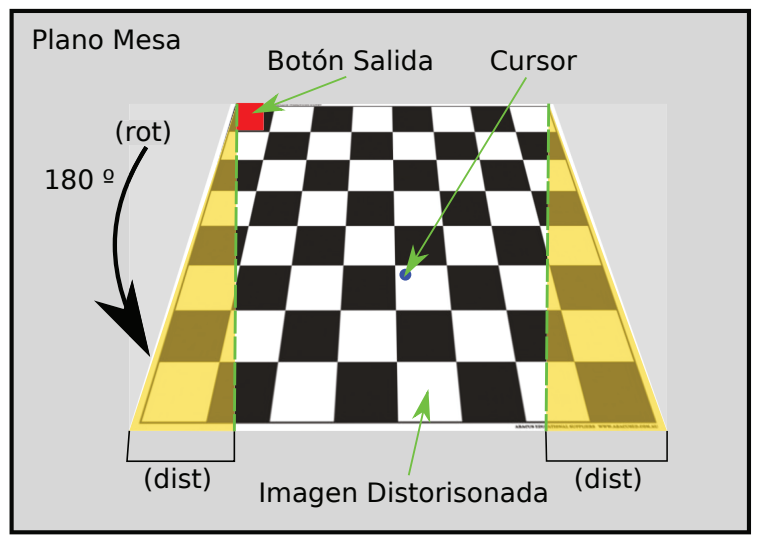

Figura 3: Las imágenes mostradas por el sistema se proyectan sobre una mesa como paralelogramos. Estas deben ser corregidas para eliminar la distorsión (dist) y rotadas $180^{\circ}$ (rot), además de añadírseles el botón de salida y el cursor. 
pen mouse como elemento característico que el jugador utiliza para interactuar con el juego, siendo necesario realizar el tracking de su posición a lo largo de la partida.

Este tipo de dispositivo ha sido escogido puesto que posee las funciones completas de un ratón de ordenador, esto es, botones y puntero infrarrojo, así como por su fácil detección con algoritmos de visión por computador dado su forma y color distintivos. Los botones permiten al usuario, por ejemplo, seleccionar, arrastrar y soltar elementos pertenecientes al juego, mientras que el puntero infrarrojo permite estimar el movimiento de este. No obstante, para poder ofrecer una mayor inmersión en el juego, se necesita estimar la posición global del pen mouse con respecto al tablero de juego proyectado y no solo su movimiento relativo, aspecto que no es posible obtener usando el puntero infrarrojo. Es por ello que en este trabajo se propone un sistema de percepción basado en el algoritmo Camshift [2], capaz de estimar la posición del pen mouse relativa al sistema de referencia del tablero de juego proyectado.

Con el fin de proveer de robustez al sistema de percepción, las imágenes son pre-procesadas para delimitar la detección del pen mouse al tablero de juego (evitando falsos positivos en zonas fuera del área de proyección), así como para reducir el ruido, empleando filtros estándares en visión por computador. Además diferentes restricciones referentes al color y tamaño del pen mouse son empleados para reducir el número de candidatos a analizar.

El resultado de la localización del bolígrafo señalador es un rectángulo definido sobre el plano de la imagen y que viene determinado por su posición, tamaño y orientación. Dentro de este paralelogramo se ajusta una elipse que representa la estructura básica del bolígrafo, y de la que uno de sus vértices se corresponde con el extremo del dispositivo apuntador (posición a estimar). Es de notar que dicho vértice varía según la forma de sujetar el dispositivo tal y como se observa en la Figura 4. En la imagen de la izquierda, bolígrafo sujetado con la mano izquierda, siendo su extremo el círculo verde. A la derecha, bolígrafo sujetado con la mano derecha, siendo su extremo el representado por el círculo rojo. Por esta razón se han implementado dos modos diferentes de funcionamiento atendiendo a si el usuario es diestro o zurdo.

Finalmente, cabe mencionar que se ha tenido en cuenta la situación en la que el bolígrafo desaparece de la imagen. En dicho caso se deshabilitan los botones incorporados y el cursor se mantiene en la última posición conocida. De esta manera se evitarían interacciones con el juego no deseadas
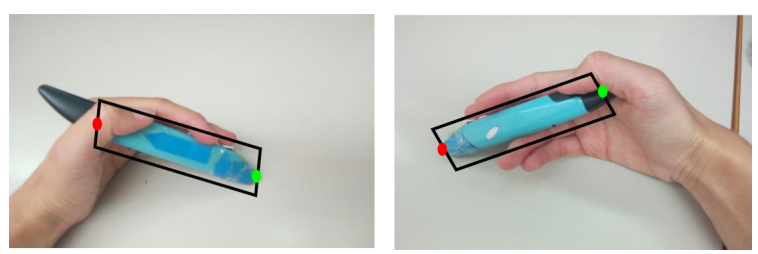

Figura 4: Localización de los vértices (representados como círculos) de la elipse que envuelve el bolígrafo encontrada mediante el algoritmo camshift.

por el usuario, por ejemplo, debido a una caída del dispositivo seleccionador.

\section{CALIBRACIÓN PROYECTOR-CÁMARA}

Debido a la posición no perpendicular tanto del proyector como de la cámara con respecto al plano de la mesa donde se proyecta el juego, se produce una distorsión en ambos elementos que debe ser corregida. En relación al proyector, esta distorsión se traduce en un aspecto trapezoidal de la imagen proyectada (véase Figura 5), mientras que para el caso de la cámara esta distorsión afecta a la imagen tomada. Esta cámara es utilizada por el sistema de percepción comentado en la Sección 3.2 y su calibración es necesaria para la correcta estimación en la posición del dispositivo señalador. Es necesario destacar que la calibración de la cámara depende de la del proyector (ya que esta observa la imagen proyectada), por lo que la calibración del proyector debe ser previa a la de la cámara.

\subsection{Calibración del Proyector}

El objetivo final es encontrar la transformación proyectiva (homografía) que aplicada a cualquier imagen a mostrar por el proyector, genere sobre el plano de la mesa una imagen sin distorsión (cuadrada/rectangular).

La principal complejidad de esta calibración radica en la falta de sensores capaces de medir la distorsión provocada por el proyector. Para solventar esto, se ha optado por utilizar una cámara situada perpendicularmente al plano de la mesa. Dicha cámara captará la proyección de la imagen sobre la mesa con la distorsión incluida. La relación entre la imagen que ha sido proyectada y la imagen captada por esta cámara proporciona la transformación proyectiva buscada. Dado que se trabaja con imágenes, dicha transformación relacionará los píxeles de la primera imagen con los de la segunda. Usualmente este tipo de transformaciones se definen por una matriz que genera la correspondencia entre un píxel de la imagen origen $(x, y)$ y un píxel en la imagen transformada 


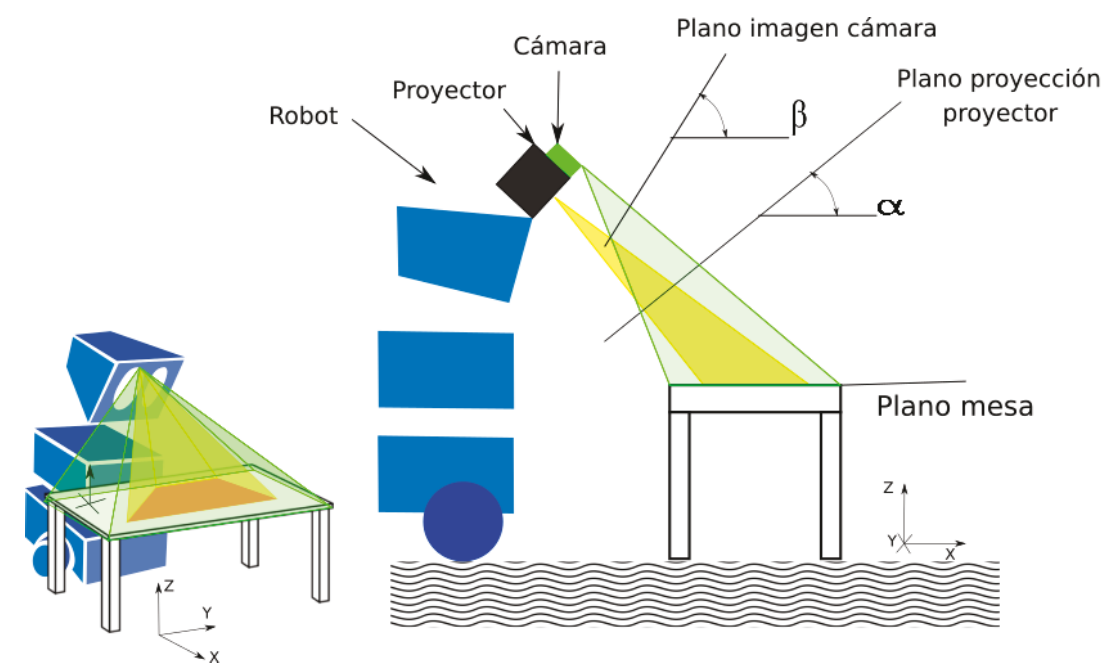

(1)

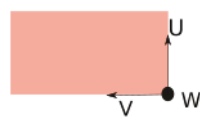

(2)
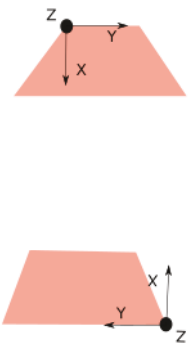

Figura 5: Diferencias angulares entre el plano de proyección y el de la mesa $(\alpha)$, y entre este y el plano de la imagen de la cámara $(\beta)$. Estas diferencias son las causantes de que la imagen a mostrar (1) adquiera una distorsión al ser proyectada sobre el plano de la mesa (2), y de la deformación en la imagen capturada por la cámara (3), respectivamente.

$\left(x^{\prime}, y^{\prime}\right)$.

$$
\left[\begin{array}{l}
x^{\prime} \\
y^{\prime} \\
z^{\prime}
\end{array}\right]=\left[\begin{array}{lll}
H_{11} & H_{12} & H_{13} \\
H_{21} & H_{22} & H_{23} \\
H_{31} & H_{32} & H_{33}
\end{array}\right]\left[\begin{array}{l}
x \\
y \\
1
\end{array}\right]
$$

Es necesario añadir que las transformaciones de este tipo son homogéneas, exigiendo que $z^{\prime}=1$. El proceso de calibración requiere establecer al menos cuatro correspondencias de puntos entre las dos imágenes. Para ello, se ha escogido una imagen patrón con cuatro puntos distribuidos simétricamente. Dado que la posición de estos puntos se conoce en la imagen a proyectar, se requiere encontrar esos mismos puntos en la imagen captada por la cámara situada en la cenital al plano de la mesa. Para ello, se hará uso del algoritmo de Hough para círculos, eliminando previamente el ruido inherente a la imagen y comenzado la búsqueda de los círculos en el centro de la misma y ampliando la ventana de examen en caso de no encontrarlos. Una vez las correspondencias han sido establecidas se calcula la matriz de transformación $H$ (veáse Ecuación (1)).

Como puede observarse en la Figura 6, la matriz $H$ transforma la imagen a mostrar de forma que al ser proyectada sea apreciada por el usuario sin distorsión. Generalmente esta transformación produce una imagen deformada de mayor tamaño y trasladada respecto del área que se proyecta. Es necesario, por tanto, ajustarla a dicha área de forma que la imagen completa sea mostrada.

\subsection{Calibración de la Cámara}

Análogamente a lo que sucede con el proyector, la posición de la cámara respecto de la mesa provo- ca una distorsión en la imagen tomada por esta. El objetivo de calibrar la cámara es obtener de la imagen una sección que se corresponda con el tablero proyectado sin deformación.

Para ello, primeramente es necesario proyectar la imagen patrón utilizada anteriormente con la distorsión del proyector corregida. Esta proyección posee un aspecto rectangular sobre la mesa, que aparecerá distorsionado en la imagen tomada por la cámara. El procedimiento y la teoría utilizados son lo mismos que aquellos seguidos durante la calibración del proyector. Conociendo la imagen patrón y sus puntos característicos, es necesario estimar dichos puntos en la imagen tomada para obtener una matriz de homografía que los relacione. Los círculos son localizados siguiendo el mismo procedimiento que durante la corrección del proyector. En este caso, la matriz transformaría la imagen capturada por la cámara en la imagen patrón.

\section{MOTOR DE JUEGOS}

En la presente Sección se explica el motor de juegos implementado en este proyecto, definiendo las dos etapas fundamentales de este: el menú y la ejecución de un juego. La primera ofrece al usuario una selección de juegos a los que poder jugar, mientras que en la segunda etapa, el juego escogido en el menú previo es lanzado para que la partida pueda comenzar. 


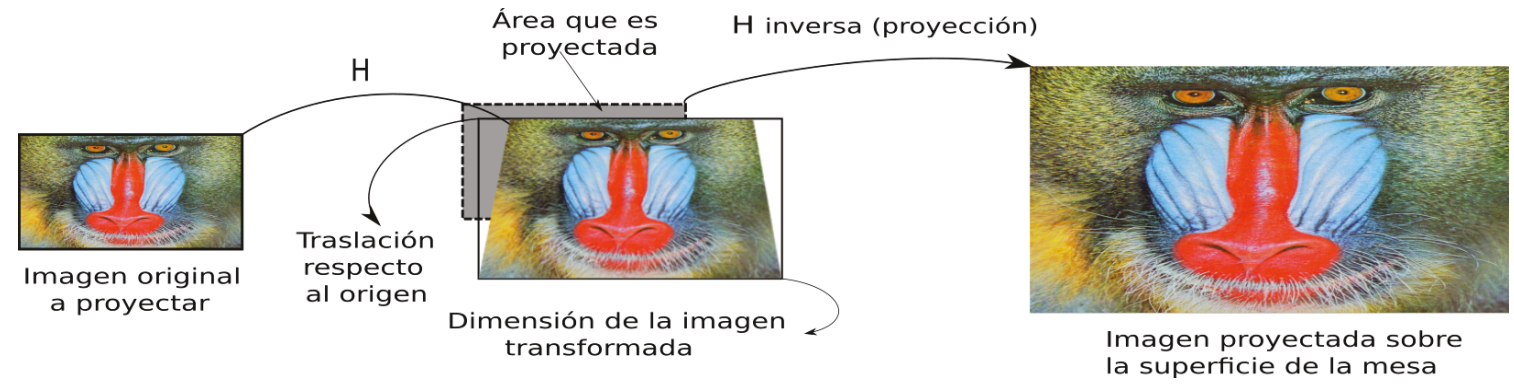

Figura 6: Corrección de una imagen mediante la matriz de transformación $H$, e ilustración de los efectos de traslación y re-dimensión asociados. La posterior proyección de esta imagen rectificada genera una imagen sin distorsión sobre la superficie de la mesa.

\subsection{Menú de Juegos}

Una importante característica de la plataforma desarrollada es la diversidad de juegos de mesa que el usuario tiene a su disposición. Con el fin de exponer dicha oferta al usuario, se ha creado una etapa previa donde se proyecta el menú con todas las opciones disponibles. En cada caso, el usuario selecciona el juego deseado colocando el pen mouse sobre el icono del mismo, y pulsado el botón izquierdo. Es necesario comentar que, durante la proyección del menú, el botón de salida da por finalizada la sesión de juegos, cerrando el menú y comandando al robot a alejarse de la mesa de juegos.

Desde un punto de vista de la implementación, y atendiendo a la arquitectura ROS empleada, el menú ha sido programado como un action server. Esto permite tener control sobre las acciones del usuario (selección de un juego o cierre de la aplicación), así como implementar ciertas opciones de seguridad y robustez, como es el cerrar automáticamente el juego tras un cierto time-out para evitar situaciones en las que el usuario se ausente.

\subsection{Lanzamiento del Juego}

Una vez el usuario selecciona el juego deseado, este se comienza a proyectar sobre la mesa y el sistema de percepción empieza a detectar los movimientos del usuario en relación a las piezas del juego.

Cabe mencionar que el juego seleccionado se ejecuta en el PC embebido en el robot. Con el objetivo de proyectar únicamente la sección de la pantalla correspondiente al juego, y no mostrar al usuario el escritorio completo, se ha implementado un nodo adicional denominado nodo de grabación que permite capturar una sección concreta de la pantalla del PC para ser posteriormente proyectada. Este nodo ROS publica las imágenes capturadas en un topic para ponerlas a disposición del nodo de proyección (véase Sección 3). En relación a este proceso, la posición del bolígrafo, estimada por el sistema de percepción, debe ser re-escalada aten- diendo a las dimensiones de la ventana del juego con respecto a las del display del robot.

Como en el caso del menú, la ejecución de cada juego particular se gestiona a través de un action server. Este es ejecutado al seleccionar un juego de entre los ofrecidos en el menú, y se mantiene activo durante toda la partida. Dicha partida finaliza cuando el usuario selecciona el botón de salida (véase Sección 3.1). Esta acción detiene el sistema de percepción y la proyección del juego, retornando a la presentación del menú.

\section{MOVIMIENTO DEL ROBOT}

El sistema propuesto se fundamenta en el uso de un robot móvil y en la mejora de su interacción con un usuario a través de un sistema que le permita jugar a juegos de mesa. Una vez presentado el sistema de juegos y las complicaciones técnicas relacionadas con la proyección del mismo, en esta sección se describe los componentes de navegación empleados para permitir al robot desplazarse por el entorno de forma segura y aproximarse a la mesa en la que se llevará a cabo la partida.

La navegación del robot, incluyendo en esta tarea la localización en un mapa conocido, la planificación del camino y la evitación de obstáculos dinámicos, ha sido implementada en base al stack de navegación de ROS. Más concretamente se emplean los siguientes paquetes:

- gmapping/map_server: para el mapeado del entorno usando técnicas de SLAM basado en laser 2D. El mapa de ocupación generado, que será empleado para la localización y planificación global del camino, es gestionado por el paquete map_server.

- amcl: sistema de localización probabilístico basado en filtro de partículas.

- move_base: para la planificación global/local del camino que el robot debe seguir 
desde su localización actual hasta la mesa de juego.

Un vez el robot ha navegado hasta una posición cercana a la mesa de juego, este debe aproximarse a la mesa para poder proyectar correctamente. No obstante, los algoritmos de navegación reactiva empleados evitan que el robot se aproxime demasiado a los obstáculos (para evitar colisiones), por lo se hace necesario implementar un sistema independiente para aproximar al robot a la mesa sobre la cual proyectar. De la misma forma, una vez acabada la sesión de juegos, el robot debe alejarse de la mesa antes de activar los algoritmos de navegación, evitando así colisiones indeseadas y mejorando la robustez del sistema.

Ambas tareas de aproximación y alejamiento se han implementado como ROS action servers, publicando comandos de velocidad directamente a la base robótica. Para la aproximación, el sistema de percepción introducido en la Sección 3.2 es usado para detectar el borde de la mesa mediante el método de Hough para líneas. Tras filtrar y seleccionar las líneas candidatas a ser el borde de la mesa, se selecciona aquella que mayor similitud guarde con la posición final deseada (configurable por parámetro). En cada iteración, el sistema de aproximación envía los comandos de velocidad estimados para conseguir que el borde de la mesa quede en la posición establecida (en el sistema de la cámara).

\section{PRUEBA DE CONCEPTO}

Con el fin de validar el funcionamiento del sistema propuesto, se ha realizado una prueba de concepto donde uno de los robots del laboratorio MAPIR ${ }^{1}$ equipado con el proyector (YG300 LCD Mini Proyector) y la webcam (Creative Live! Cam Sync HD 720p), ejecutaba todos las tareas involucradas en este proyecto para ofrecer jugar una partida a un usuario. Para la interacción robot-usuario, se ha utilizado un pen-mouse inalámbrico de la marca $\operatorname{Sodial}(R)$. Proyector y cámara han sido colocados a una altura aproximada de 0.8 y $0.85 \mathrm{~m}$, respectivamente. Esta distancia es la mínima requerida por el proyector para mostrar una imagen nítida, y le proporciona a la cámara un amplio rango de visión. La Figura 7 muestra un fotografía del robot donde se destacan los diferentes componentes del sistema. Puede observarse como una sección del display incorporado en el robot (naranja) es mostrada mediante el proyector (rojo). El sistema compuesto por el proyector y el sistema de percepción aparece en rosa, así como el dispositivo seleccionador comentado (verde).

\footnotetext{
${ }^{1}$ http://mapir.uma.es
}

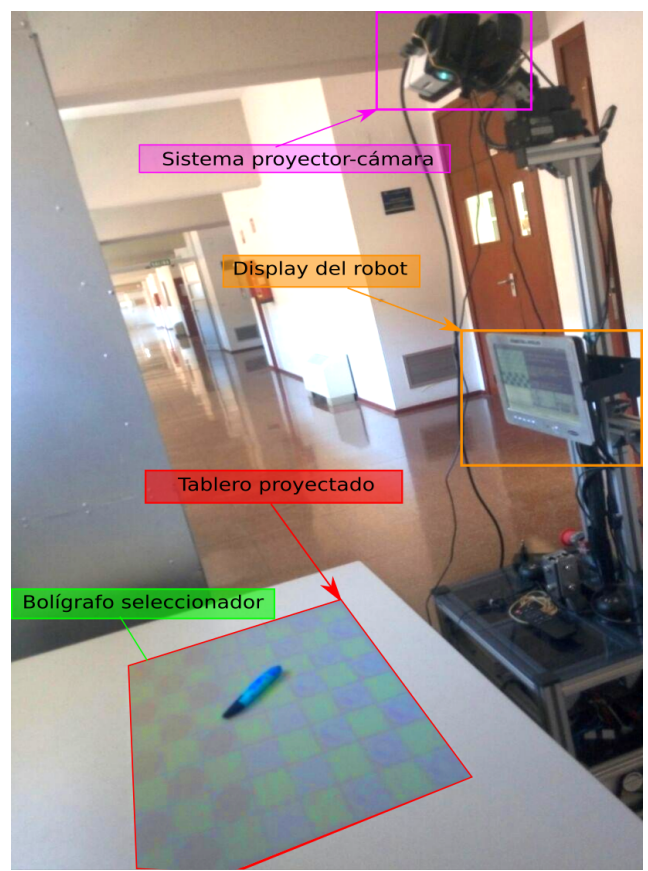

Figura 7: Fotografía del robot móvil Rhodon durante una partida de damas, remarcando los principales elementos del sistema.

Además de los dispositivos propios de este proyecto, se hace uso de los sensores y actuadores ya incorporados en el robot para las tareas de navegación. Igualmente, y sobre todo para tareas de testeo, se hace uso del display incorporado en el robot, que se utilizará como pantalla principal.

En relación a la selección de juegos ofertados en la fase del menú (véase Sección 5.1), y dada las características del proyector empleado, se han limitado las opciones expuestas en el menú a 6 juegos. Es necesario especificar que dichos juegos tiene como único requisito el poder ser jugados únicamente empleando un dispositivo apuntador, como ocurre en el caso de las damas o el ajedrez.

\section{CONCLUSIONES Y TRABAJOS FUTUROS}

En este proyecto se ha dotado a un robot móvil de la capacidad de ofrecer y participar en juegos de mesa. Un énfasis especial ha sido puesto en la facilidad de uso del sistema y en la forma de interactuar con el usuario, manteniendo la distribución clásica de este tipo de juegos. Para ello, se ha hecho uso de un proyector con el fin de recrear el juego sobre una mesa, y de un sistema de percepción que permite estimar los movimientos del usuario. La calibración de los sistemas de proyección y percepción ha sido contemplada, calculando las homografías correspondientes para corregir las deformaciones derivadas de las posiciones relativas de estos sistemas con respecto a la superficie 
de juego. Finalmente, el sistema de navegación implementado y el módulo de aproximación a la mesa de proyección han sido descritos, y el sistema ha sido validado con un experimento real en un entorno de laboratorio.

Como trabajo futuro se contempla el desarrollo de un sistema de calibración automático para el conjunto proyector-cámara, así como una mejora de la interacción por voz, permitiendo al usuario controlar la plataforma de juegos mediante comandos de voz.

\section{Agradecimientos}

Trabajo realizado en el marco del proyecto WISER (DPI2017-84827-R), financiado por el Ministerio de Ciencia e Innovación contando con fondos del Fondo Europeo de Desarrollo Regional (FEDER) y a la Beca de Colaboración del Ministerio de Educación, Cultura y Deporte (998142).

\section{English summary}

\section{LETS PLAY! IMPROVEMENT OF HUMAN-ROBOT INTERACTION THROUGH BOARD GAMES}

\section{Abstract}

In the context of a service or assistance robot, and in relation to its interaction with the user, this work presents a system that allows the robot to offer and carry out board games. The proposed system, which seeks to expand the range of activities that the robot can share with the user, focuses on offering an experience as similar as possible to a traditional game between two people. To do this, the game is projected on a table by means of a small projector mounted on the robot, and a visual perception system is used to detect the movements of the user. The system is especially interesting for care robots in residential settings with elderly people, allowing the user to carry out different activities beneficial for health (memory, reflexes, coordination, etc.). To validate the proposed system, a practical case is presented with a real robot in a laboratory environment.

Keywords: HRI, human-robot interaction, board games, projective transformation, ROS

\section{Referencias}

[1] J. M. C. Bordería, R. P. Puente, R. B. i Guillem, M. F. Cabrera, and M. C. Ferrer. Juegos de mesa y personas mayores: la importancia de nuevos diseños. Revista de biomecánica, (42):11-12, 2004.
[2] G. Bradski. Real time face and object tracking as a component of a perceptual user interface. In IEEE Workshop on Applications of Computer Vision. WACV'98., 1998.

[3] S. Coradeschi, A. Cesta, G. Cortellessa, L. Coraci, C. Galindo, J. Gonzalez-Jimenez, L. Karlsson, A. Forsberg, F. S., F. Furfari, A. Loufti, A. Orlandini, F. Palumbo, F. Pecora, A. Stimec, S. V. Rump, J. Ulberg, and B. Otslund. GiraffPlus: A System for Monitoring Activities and Physiological Parameters and Promoting Social Interaction for Elderly, pages 261-271. Human-Computer Systems Interaction: Backgrounds and Applications 3. Springer, 2014.

[4] H. M. Gross, S. Mueller, C. Schroeter, M. Volkhardt, A. Scheidig, K. Debes, K. Richter, and N. Doering. Robot companion for domestic health assistance: Implementation, test and case study under everyday conditions in private apartments. pages 5992-5999, Sept 2015.

[5] J. Monroy, J. R. Ruiz-Sarmiento, F.-A. Moreno, and J. Gonzalez-Jimenez. Towards a Semantic Gas Source Localization under Uncertainty. Springer, Cham, 2018.

[6] A. Orlandini, A. Kristoffersson, L. Almquist, P. Björkman, A. Cesta, G. Cortellessa, C. Galindo, J. Gonzalez-Jimenez, K. Gustafsson, A. Kiselev, A. Loufti, F. Melendez-Fernandez, M. Nilsson, L. Odens Hedman, E. Odontidou, J. R. Ruiz-Sarmiento, M. Scherlund, L. Tiberio, S. V. Rump, and S. Coradeschi. Excite project: A review of forty-two months of robotic telepresence technology evolution. Presence: Teleoperators and Virtual Environments, 2017.

[7] M. Quigley, K. Conley, B. Gerkey, J. Faust, T. Foote, J. Leibs, R. Wheeler, and A. Y. Ng. Ros: an open-source robot operating system. In ICRA workshop on open source software, volume 3, page 5. Kobe, Japan, 2009.

[8] C. b. G. Riva. Teresa: Socially intelligent robot as window to the world. Cyberpsychology, Behavior, and Social Networking, 20(5):343343, 2017.

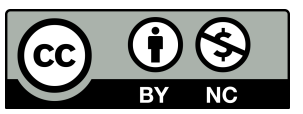

(c) 2018 by the authors. Submitted for possible open access publication under the terms and conditions of the Creative Commons Attribution CC-BY-NC 3.0 license (http://creativecommons.org/licenses/by-nc/3.0/). 